\title{
Detection of micrometastases in lymph nodes using reverse transcription polymerase chain reaction (RT-PCR) for cytokeratin 20 (CK-20) - a pilot study
}

\author{
S R E Wijesuriya ${ }^{1}$, K G R Kuruppuarachchi ${ }^{2}$, A Weerasinghe ${ }^{3}$, J Hewavisenthi ${ }^{4}$, K I Deen $^{1}$
}

(Index words: neoadjuvant therapy, rectal cancer, lymph nodes)

\begin{abstract}
Objectives The aim of the study was to detect micrometastases in lymph nodes in patients with rectal cancer following neoadjuvant therapy, staged node negative by routine histology.

Patients and setting Mesenteric lymph nodes from patients who have undergone neoadjuvant therapy for rectal cancer were harvested during surgery. Nodes were bisected and one half was sent for haematoxylin and eosin (H\&E) staining and evaluated by a single pathologist. The other half was examined for CK20 by RT-PCR. The technique was validated by testing mesenteric lymph nodes with known metastases and nodes from patients without cancer. Twenty one lymph nodes from 6 patients (median age 46 years, range 2555) which were negative for tumour deposits by H\&E stain were assessed for micro-metastases.
\end{abstract}

Results All 21 nodes which were histologically negative for metastases were positive for micrometastases. Two nodes with known metastases were positive for CK20 and 3 nodes from non cancer patients were negative for CK20.

Conclusions Detection of $\mathrm{CK} 20$ is accurate in identification of rectal cancer micro-metastasing to lymph nodes. Assessment of nodes by $\mathrm{H}$ \& $\mathrm{E}$ histology risks under staging.

Ceylon Medical Journal 2010; 55: 77-9

\section{Introduction}

Managing locally advanced rectal cancer is challenging. One main issue is the high incidence of local recurrence following resection. Out of many recent advances in minimising local recurrence, neoadjuvant therapy seems to be a promising strategy [1], especially in locally advanced cases (T3 and T4). However, neoadjuvant therapy also has several draw backs. These include adverse reactions to irradiation and inaccurate staging, especially with regard to assessment of lymph nodes [2]. Inaccurate staging may occur as a result of tumour down staging, fibrosis and significantly lower yield of lymph nodes and their smaller size [2]. Such situations may require more sensitive techniques in staging instead of currently used hematoxylin and eosin (H\&E) staining. CK20 is a cytokeratin known to be specific to colonic epithelium which may help detection of rectal cancer metastases in lymph nodes. Use of a technique such as reverse transcription polymerase chain reaction (RT-PCR) using CK 20 primers may be a more appropriate method in patients with rectal cancer who have been subjected to neoadjuvant therapy [3].

The aim of this study was to detect micrometastases in lymph nodes in patients with rectal cancer following neoadjuvant therapy, staged node negative by routine histology.

\section{Methods}

Patients were recruited to the study from a surgical clinic at the North Colombo Teaching Hospital, Ragama, Sri Lanka. The eligibility criteria to receive neoadjuvant therapy included T3 or T4 adenocarcinoma of the rectum staged by contrast CT or MRI, less than 75 years of age and not previously treated with radiotherapy or chemotherapy. Informed written consent was obtained from all patients prior to enrolment. Assessment consisted of a clinical assessment followed by baseline haematological and radiological evaluation for staging the disease and fitness for operation.

Neoadjuvant therapy consisted of a high dose radiotherapy protocol that employed a total radiation dose of 4500 cGy in 25 fractions over five weeks. External beam irradiation was delivered via two portals, and the field of irradiation included the rectal tumour including the anal canal, the mesorectum and pelvic lymph nodes below the fifth lumbar vertebra. 5 fluoro-uracil was infused intravenously (375mg/m²/day) during the first and last weeks of irradiation.

The operation was undertaken four to six weeks after completion of neoadjuvant therapy. The surgical procedures that were performed were either anterior

${ }^{1}$ Department of Surgery, University of Kelaniya, Sri Lanka, ${ }^{2}$ Department of Molecular Biology, Medical Research Institute, Colombo, Sri Lanka, ${ }^{3}$ Department of Physiology, and ${ }^{4}$ Department of Pathology, University of Kelaniya, Sri Lanka.

Correspondence: SREW, e-mail <ruwanwije@hotmail.com>. Received 15 March and accepted 5 June 2010. Competing interests: none declared. 
resection or abdominoperineal resection with total mesorectal excision. All surgical procedures were performed by a single colorectal surgeon and the excised specimens were evaluated by one pathologist. During the surgical procedure, all precautions were observed to avoid contamination of operating field by skin scales and spillage of colonocytes during resection. Lymph nodes were subsequently dissected by a single author for evaluation. Dissection was along the superior rectal vessels up to the apex of the mesentery without employment of fat cleansing chemical agents.

Each lymph node was bisected and one half was fixed in formalin and the other half in Isogen ${ }^{\circledR}$. Each specimen was stored in separate labelled containers. Paraffin embedded sections of lymph nodes were stained with H\&E and were evaluated for tumour metastases. Twenty one lymph nodes from 6 patients (median age 46 years, range 25-55) which did not have tumour metastases by H\&E staining technique were included in the study. Subsequently the corresponding halves of the lymph nodes were evaluated for CK20 using RT-PCR. In addition, the corresponding half of two lymph nodes found to have tumour metastases by $\mathrm{H} \& \mathrm{E}$ staining, were also evaluated by RT-PCR as positive controls. Three lymph nodes from patients with ulcerative colitis without cancer were included as negative controls.

\section{Technique of RT-PCR}

All specimens which were immersed in Isogen were frozen immediately at $-70^{\circ} \mathrm{C}$. During the PCR assay, cytoplasmic RNA of tumour cells within lymph nodes were extracted by the acid-guanidinium-thiocyanate-phenolchloroform method [4]. Extracted RNA was then dissolved in $20 \mu \mathrm{l}$ of RNase free water. RNA was quantified by spectrometry at $260 \mathrm{~nm}$ and $280 \mathrm{~nm}$. RNA was subsequently reverse transcribed in a mixture containing $2 \mu \mathrm{l}$, RT buffer, $2.0 \mathrm{mmol} / \mathrm{l}$ of dithiothreitol (DTT), Moloney murine leukemia virus reverse transcriptase (MMLV) $1 \mu 1$, $4 \mu 1 \mathrm{RNase}$ inhibitor, $2.5 \mu \mathrm{mol} / 1 \mathrm{random}$ hexamer and 0.5 $\mathrm{mmol} / \mathrm{l}$ deoxynucleotide triphosphate (dNTP). CK 20 primers were used for the PCR and the mixture was incubated for 60 minutes at $37^{\circ} \mathrm{C}$, and then reverse transcriptase was inactivated for 10 minutes at $95^{\circ} \mathrm{C}$.

Expression of a house-keeping gene, $\beta$-actin, was also subjected to the same protocol simultaneously as a marker of internal quality control. The PCR reaction mixture was electrophoretically separated on a $2 \%$ agarose gel containing ethidium bromide and visualised under ultraviolet light. A clear band which corresponded with marker of 298bp was sought. Presence of the described band for CK 20 was considered positive.

\section{Results}

All 21 nodes harvested from patients with rectal cancer that did not have visible tumour metastases by H\&E, were positive by CK20 assay. The two nodes which were harvested and used as positive controls from patients who had lymph node metastases were also positive for CK20, but the three nodes from non-cancer patients were negative for CK20 (Table 1).

Table 1. Specimens analysed by RT-PCR assay

\begin{tabular}{|c|c|c|c|}
\hline Specimen & $\begin{array}{l}\text { Number of } \\
\text { lymph nodes }\end{array}$ & $\begin{array}{c}\text { Number positive } \\
\text { by H \& E stain }\end{array}$ & $\begin{array}{c}\text { Number positive } \\
\text { for CK20 }\end{array}$ \\
\hline $\begin{array}{l}\text { Lymph nodes without } \\
\text { metastases harvested } \\
\text { from rectal cancer specimens } \\
\text { (Test nodes) }\end{array}$ & 21 & 0 & 21 \\
\hline $\begin{array}{l}\text { Lymph nodes with metastases } \\
\text { harvested from rectal cancer } \\
\text { specimens } \\
\text { (Positive control) }\end{array}$ & 02 & 02 & 02 \\
\hline $\begin{array}{l}\text { Lymph nodes harvested from } \\
\text { Non cancer specimens } \\
\text { (Negative control) }\end{array}$ & 03 & 0 & 0 \\
\hline
\end{tabular}




\section{Discussion}

Evaluation of mesenteric lymph nodes in rectal cancer for micrometastases may have a direct effect on surgical practice. Evidence is emerging regarding the association between micrometastases in lymph nodes and poorer outcome following resection of rectal cancer, especially when it is extensive and involving distant nodes [5,6,7,8]. Post-operative chemotherapy may benefit this group of patients.

According to the results of our study tumour stage had been upstaged by $100 \%$. There seems to be a discrepancy between these results and results of other studies where upstaging was less than fifty percent $[6,9,10]$. One or more of following hypotheses could explain this difference.

The main difference between this study and quoted studies is that all patients in this sample had undergone preoperative chemo-irradiation. High dose neoadjuvant therapy for a period of five weeks followed by a further interval of 4-6 weeks prior to surgical resection allows time for regression of mesenteric lymph nodes [1]. In addition lymph nodes following exposure to radiation seems to have altered architecture. As a result metastatic tumour deposits may not be easily detected by routine $\mathrm{H} \& \mathrm{E}$ staining. Therefore in these specimens we may actually be detecting regressed macrometastases in addition to micrometastases which otherwise would have been missed by H\&E staining.

An alternative explanation could be that all patients in this study had locally advanced tumours (T3 and T4) compared with other reports [10] and micrometastases can be assumed to be more likely in advanced tumour stages.

Further studies with larger samples should be performed and compared with specimens which had not been previously irradiated to determine the place of detection of micrometastases in rectal cancer following neoadjuvant therapy.

Despite the fact that neoadjuvant therapy down stages rectal cancer and reduces the number and size of lymph nodes, it seems that neoadjuvant therapy has actually failed to sterilize lymph nodes. Employment of a technique such as immunohistochemistry or RT-PCR for CK20 may have an important role in staging of rectal cancer especially following neoadjuvant therapy. For accurate staging of rectal cancer it is believed that at least 10-12 lymph nodes should be examined. However as neoadjuvant therapy results in significantly lower lymph node yield, there is a risk of under staging the disease. Hence we believe that detection of CK20 by RT-PCR should be part of routine staging of rectal cancer following neoadjuvant therapy to overcome the limits of histological staging.

\section{Acknowledgements}

Financial grants were provided by National Research Council of Sri Lanka.

\section{References}

1. Bosset JF, Pelissier EP, Maniion G, et al. Plea for a preoperative adjuvant approach in the management of rectal cancer. International Journal of Radiation Oncology Biology Physics 1994; 29: 205-8.

2. Wijesuriya RE, Deen KI, Hewavisenthi J, Balawardana J, Perera M. Neoadjuvant therapy for rectal cancer downstages the tumour but reduces lymph node harvest significantly. Surgery Today 2005; 35: 442-5.

3. Liu Z, Ye X, Bi W, Wang M, Li Y, Chen T. Detection of occult metastases in lymph nodes from patients with colorectal carcinoma by reverse transcriptase-polymerase chain reaction. Chinese Medical Journal 2002; 115: 529-31.

4. Chomezynski P, Sacchi N. Single-step method of RNA isolation by acid guanidinium thiocyanate-phenolchloroform extraction. Analytical Biochemistry 1987; 162: 156-9.

5. Merrie AE, van Rij AM, Dennett ER, et al. Prognostic significance of occult metastases in colon cancer. Diseases of the Colon and Rectum 2003; 46: 221-31.

6. Noura S, Yamamoto H, Ohnishi T, et al. Comparative detection of lymph node micrometastases of stage II colorectal cancer by reverse transcriptase polymerase chain reaction and immunohistochemistry. Journal of Clinical Oncology 2002; 20: 4232-41.

7. Yasuda K, Adachi Y, Shiraishi N, et al. Pattern of lymph node micrometastasis and prognosis of patients with colorectal cancer. Annals of Surgical Oncology 2001; 8: 300-4.

8. Miyake Y, Yamamoto H, Fujiwara Y, et al. Extensive micrometastases to lymph nodes as a marker for rapid recurrence of colorectal cancer: a study of lymphatic mapping. Clinical Cancer Research 2001; 7: 1350-7.

9. Lassmann S, Bauer M, Rosenberg R, et al. Identification of occult tumor cells in node negative lymph nodes of colorectal cancer patients by cytokeratin 20 gene and protein expression. International Journal of Colorectal Disease 2004; 19: 87-94.

10. Kronberg U, López-Kostner F, Soto G, et al. Detection of lymphatic micrometastases in patients with stages I and II colorectal cancer: impact on five-year survival. Diseases of the Colon and Rectum 2004; 47: 1151-7. 